\title{
The impact of therapeutics on mortality in hospitalised patients with COVID-19: systematic review and meta-analyses informing the European Respiratory Society living guideline
}

\author{
Megan L. Crichton ${ }^{1}$, Pieter C. Goeminne ${ }^{2}$, Krizia Tuand $\mathbb{1}^{3}$, Thomas Vandendriessche ${ }^{3}$, Thomy Tonia ${ }^{4}$, \\ Nicolas Roche ${ }^{5}$ and James D. Chalmers ${ }^{1}$ on behalf of the European Respiratory Society COVID-19 Task Force ${ }^{6}$ \\ ${ }^{1}$ School of Medicine, University of Dundee, Dundee, UK. ${ }^{2}$ Dept of Respiratory Medicine, AZ Nikolaas, Sint-Niklaas, Belgium. ${ }^{3} \mathrm{KU}$ Leuven \\ Libraries - 2Bergen - Learning Centre Désiré Collen, Leuven, Belgium. ${ }^{4}$ Institute of Social and Preventive Medicine, University Bern, \\ Bern, Switzerland. ${ }^{5}$ Respiratory Medicine, Cochin Hospital, APHP Centre-University of Paris, Cochin Institute (INSERM UMR1016), Paris, \\ France. ${ }^{6} \mathrm{~A}$ list of the European Respiratory Society COVID-19 Task Force members can be found in the Acknowledgements section.
}

Corresponding author: James D. Chalmers (jchalmers@dundee.ac.uk)

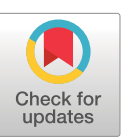

This version is distributed under the terms of the Creative Commons Attribution NonCommercial Licence 4.0. For commercial reproduction rights and permissions contact permissions@ersnet.org

This article has supplementary material available from err.ersjournals.com

Received: 16 July 2021 Accepted: 8 Sept 2021

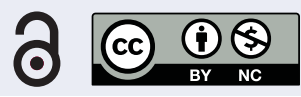

Shareable abstract (@ERSpublications)

Corticosteroids and anti-IL-6 receptor antagonist monoclonal antibodies reduce mortality in hospitalised patients with COVID-19 https://bit.ly/3k3Ax9g

Cite this article as: Crichton ML, Goeminne PC, Tuand K, et al. The impact of therapeutics on mortality in hospitalised patients with COVID-19: systematic review and meta-analyses informing the European Respiratory Society living guideline. Eur Respir Rev 2021; 30: 210171 [DOI: 10.1183/ 16000617.0171-2021].

\section{Abstract}

Hospitalised patients with coronavirus disease 2019 (COVID-19) have a high mortality rate. There are an increasing number of published randomised controlled trials for anti-inflammatory, anti-viral and other treatments. The European Respiratory Society Living Guidelines for the Management of Hospitalised Adults with COVID-19 were published recently, providing recommendations on appropriate pharmacotherapy.

Patient, Intervention, Comparator and Outcomes questions for key interventions were identified by an international panel and systematic reviews were conducted to identify randomised controlled trials meeting the inclusion criteria. The importance of end-points were rated, and mortality was identified as the key "critical” outcome for all interventions. Random-effects meta-analysis was used to pool studies and provide effect estimates for the impact of treatments on mortality.

Corticosteroids, hydroxychloroquine, azithromycin, remdesivir, anti-interleukin (IL)-6 monoclonal antibodies, colchicine, lopinavir/ritonavir and interferon- $\beta$ have been reviewed.

Our results found further evidence in support of the use of corticosteroids, particularly dexamethasone, and anti-IL-6 receptor monoclonal antibody therapy. These data support the need to identify additional therapies with beneficial effects on mortality.

\section{Introduction}

Severe acute respiratory syndrome coronavirus 2 (SARS-CoV-2) is the virus that causes coronavirus disease 2019 (COVID-19). The outbreak of this coronavirus was first identified in Wuhan (Hubei, China) in December 2019, and it was declared as pandemic by the World Health Organization in March 2020. By 27 June 2021, reported cases exceeded 180 million and its significant mortality rate (2.3\% altogether, but $\sim 10 \%$ in the elderly and $\sim 50 \%$ in the intensive care unit) [1] has led to almost 4 million deaths globally [2], making mortality a key outcome for treatment decision making.

Awaiting results from clinical trials, clinicians quickly began prescribing repurposed treatments based on their pharmacological properties, pre-clinical data (mostly from in vitro experiments) and clinical studies in other viral diseases [3]. Today, several vaccines against SARS-CoV-2 have been approved, and there are currently $>2000$ ongoing clinical trials focusing on the use and effectiveness of various therapies registered 
on clinicaltrials.gov. Some examples include antiviral agents and drugs with antiviral properties, antibiotics, anti-inflammatory drugs and antimalarial agents.

Recommendations on clinical practice for severe COVID-19 management were eagerly awaited by clinicians; however, early in the pandemic such recommendations could be based only on expert opinion, which led some scientific societies such as the European Respiratory Society (ERS) and American Thoracic Society (ATS) to use the term "guidance" rather than "guidelines" [4-6]. This was to avoid any confusion with evidence-based clinical practice guidelines developed using a robust and strict methodology such as the one proposed by the Grading of Recommendations Assessment, Development and Evaluation (GRADE) working group [7]. While the aforementioned guidance was produced by ERS/ATS taskforces [4-6], the ERS decided to initiate the preparation of evidence-based living guidelines on the management of COVID-19. The term "living guideline" refers to the objective of the taskforce to revise the recommendations whenever required following the release of new evidence with potential practical implications. The first topic addressed has been the management of hospitalised patients, which produced guidelines answering 11 Patients, Intervention, Comparison and Outcomes (PICO) questions addressing the following therapeutic options: corticosteroids, anti-interleukin (IL)-6, remdesivir, anticoagulants, hydroxychloroquine, azithromycin, hydroxychloroquine/azithromycin combination, colchicine, lopinavir/ ritonavir, interferon- $\beta$ and ventilatory support. These guidelines relied on comprehensive systematic reviews and explicit evidence-to-decision processes described in detail in the original publication [8]. For each outcome, the effect of treatment on mortality was identified as the key clinical outcome for decision making. The rapidly evolving evidence base for COVID-19 means that a large number of additional studies have been published since the first version of the ERS living guideline. In this article we present updated results of the systematic review and meta-analyses informing the ERS COVID-19 living guideline showing the impact of different COVID-19 therapeutics on mortality in hospitalised patients. It does not aim to provide additional/updated recommendations, as this will be done in future updates of the living guideline

\section{Methods}

The guideline methodology has been described in detail [8]. The objective of this systematic review and meta-analysis was to investigate the effect of nine treatment modalities on mortality in hospitalised patients with COVID-19. In the original guideline development process, no randomised trials were identified for the PICO questions addressing anticoagulation and ventilation; therefore, no randomised trials could be included for meta-analysis in this update. These topics will be addressed, along with new topics, in the next update of the living guideline. This update focused on the nine interventions for which randomised controlled studies were identified: corticosteroids, anti-IL-6 monoclonal antibodies, remdesivir, hydroxychloroquine, azithromycin, hydroxychloroquine/azithromycin combination, colchicine, lopinavir/ ritonavir and systemic interferon- $\beta$.

\section{Systematic literature review}

This update followed the same method with systematic literature searches carried out in PubMed and Embase for published peer-reviewed studies, supplemented by searches of MedRxiv for preprints until 31 May 2021 (supplementary figure S1). The systematic review and data extraction were performed by two independent reviewers (MLC and PCG) with extracted data verified and discrepancies resolved by a third independent reviewer (JDC). The original search strategies were reviewed, and Medical Subject Headings (MeSH) terms and keywords were updated where necessary by experienced librarians (KT and TV). Searches for interferon- $\beta$ and colchicine were conducted using the individual search terms plus a set of search terms for COVID-19 using PubMed and MedRxiv only. As it was expected that searches for hydroxychloroquine and azithromycin individually would capture trials in which both drugs were used in combination, no repeat searches were performed and trials were selected from the hydroxychloroquine and azithromycin search results for inclusion in the analysis. The search terms used for each PICO question are reported in the original guideline [8]. In addition, the authors identified and searched relevant published systematic reviews and meta-analyses for trials not identified by the relevant searches. The authors also searched reference lists of relevant trials and included any new trials published following the original search dates which became known to authors during writing of the article, to ensure data were as up to date as possible.

\section{Inclusion criteria}

Studies were eligible for inclusion if they were randomised clinical trials according to the International Committee of Medical Journal Editors definition (a research study that prospectively assigns a group of people to an intervention, with or without concurrent comparison or control groups, to study the relationship between a health-related intervention and a health outcome); published in English; included 
hospitalised patients with COVID-19 infection; and tested one of the interventions included in the PICO questions. For the purposes of analysis, we sought trials that tested the intervention against standard care. Where studies had been identified previously by literature searches conducted prior to the publication of the first guideline, updated data were sought; for example, preprints were replaced by peer-reviewed data and preliminary reports were replaced by more definitive reports.

\section{Data extraction and statistical analysis}

Data were extracted from source publications, tabulated and entered into RevMan 5 software version 5.3 (Cochrane Collaboration). We extracted the number of enrolled patients, baseline patient demographics, details of the patient population and mortality in the intervention and control groups in each study. Data extracted were from the intention-to-treat population wherever possible. Where mortality data were reported for multiple time points, the longest duration of follow-up was used for comparison unless the primary outcome was specified as mortality in which case the time point for the primary outcome was displayed. Random-effects meta-analysis was used based on expected heterogeneity in trial designs and patient characteristics. Heterogeneity was assessed using the $\mathrm{I}^{2}$ statistic. Based on interpretations in the Cochrane Handbook, 0-30\% was considered no heterogeneity, 30-50\% was considered moderate heterogeneity, 51$75 \%$ was considered substantial heterogeneity and $76-100 \%$ considerable heterogeneity. Where heterogeneity was detected, additional analyses were performed to explore the source of the heterogeneity. Sub-analyses were performed where a significant effect on mortality was observed in the primary analysis. In the case of a primary analysis with $\mathrm{p}>0.05$, no sub-analyses were performed. $\mathrm{p}<0.05$ was considered statistically significant for all comparisons. The GRADE approach was used to assess the quality of evidence for each outcome. GRADE has four levels of evidence: very low, low, moderate and high. GRADE was implemented using GRADEpro software and was performed by three investigators (MLC, NR and JDC), supervised by an experienced methodologist (TT).

\section{Results}

The original ERS guideline for management of hospitalised patients with COVID-19 published in March 2021 was based on a literature search up until the end of February 2021. This search identified 44 eligible references from 14851 articles and provided recommendations for 11 PICO topics: corticosteroids, IL-6 receptor antagonist monoclonal antibody, hydroxychloroquine, azithromycin, hydroxychloroquine in combination with azithromycin, lopinavir/ritonavir, remdesivir, colchicine, interferon- $\beta$, anticoagulation and noninvasive ventilatory support.

The literature search for this update retrieved an additional 12960 articles between PubMed and Embase across nine searches (anticoagulation and noninvasive ventilatory support are not included). After removal of duplicates and exclusion of citations that did not meet the established inclusion criteria while screening titles and abstracts, a total of 15 new trials meeting the eligibility criteria were identified. A search of the MedRxiv database identified a further five preprints. Together, these searches have identified 20 new references which were included in this update and were pooled with data extracted from the 44 studies identified in the searches up to February 2021.

\section{Corticosteroids}

We identified nine studies [9-17] with 7766 subjects, including three additional studies [13, 15, 17] following publication of the guidelines. Overall, mortality in the treatment groups was 714 (25.6\%) out of 2793, and in the control groups was 1365 (27.4\%) out of 4973. The pooled effect estimate showed OR 0.80 (95\% CI $0.64-1.01, \mathrm{p}=0.06$ ), indicating a mortality benefit that narrowly failed to meet the pre-specified level of statistical significance. 2104 (75.3\%) out of 2793 patients receiving corticosteroids and 4321 (86.9\%) out of 4973 patients receiving standard care were from a single trial, the RECOVERY trial of $6 \mathrm{mg}$ daily dexamethasone. The forest plot is shown in figure 1 . There was moderate heterogeneity $\left(\mathrm{I}^{2}=30 \%\right)$. The heterogeneity was entirely resolved $\left(\mathrm{I}^{2}=0 \%\right)$ after excluding the study by EDALATIFARD et al. [9] (IRCT20200404046947N1), which showed an extreme beneficial effect (OR 0.08, 95\% CI 0.02-0.42). A sub-analysis was performed to investigate whether the beneficial effects of corticosteroids were limited to dexamethasone or extend to other corticosteroids (supplementary figure S2). Four studies investigated methylprednisolone, giving an OR 0.48 (95\% CI 0.16-1.46, p=0.02) [9, 14, 15, 17]; three trials investigated dexamethasone, showing OR 0.86 (95\% CI 0.76-0.97, $\mathrm{p}=0.01)$ [12, 13, 16]; and the final two studies investigated the use of hydrocortisone: OR 0.67 (95\% CI 0.35-1.29, p=0.23) [10, 11]. The test for subgroup analysis did not show any significant differences between groups $(p=0.46)$. In the RECOVERY trial, the treatment benefit was seen most clearly in patients requiring mechanical ventilation at randomisation (rate ratio $0.64,95 \%$ CI $0.51-0.81$ ) and patients receiving supplementary oxygen (rate ratio 0.82, 95\% CI 0.72-0.94). No benefit was observed in those not requiring oxygen (rate ratio 1.19, 95\% CI 0.92-1.55). Three studies (CoDEX, CAPE COVID and REMAP-CAP) were primarily or exclusively 


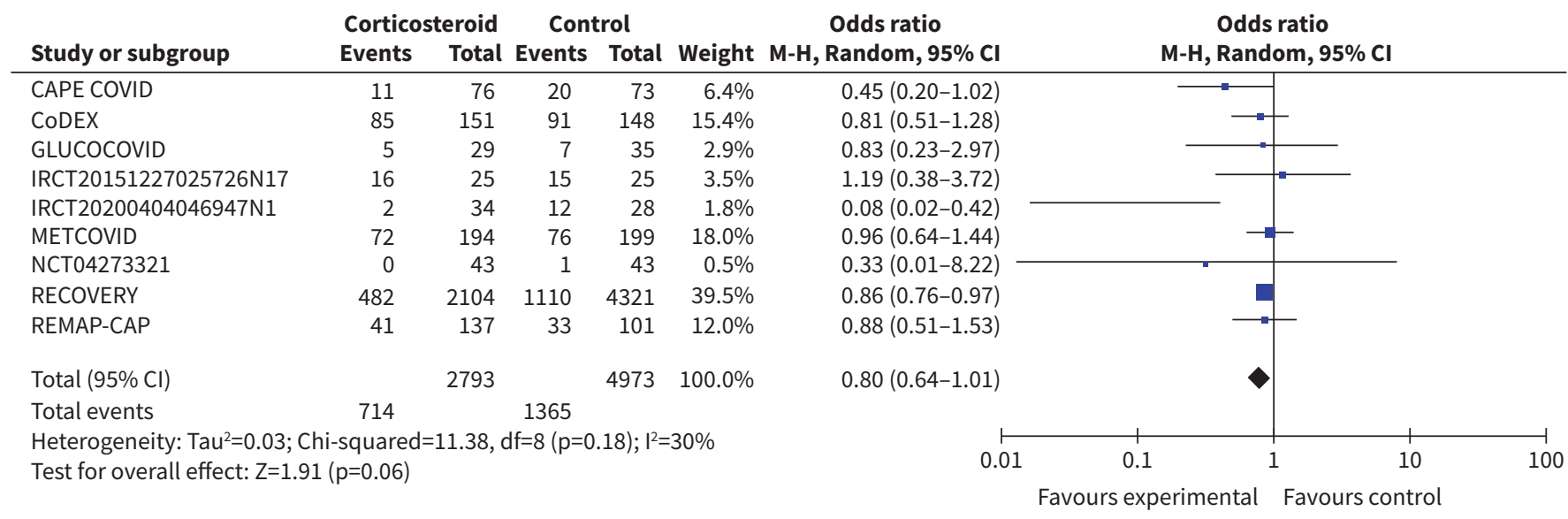

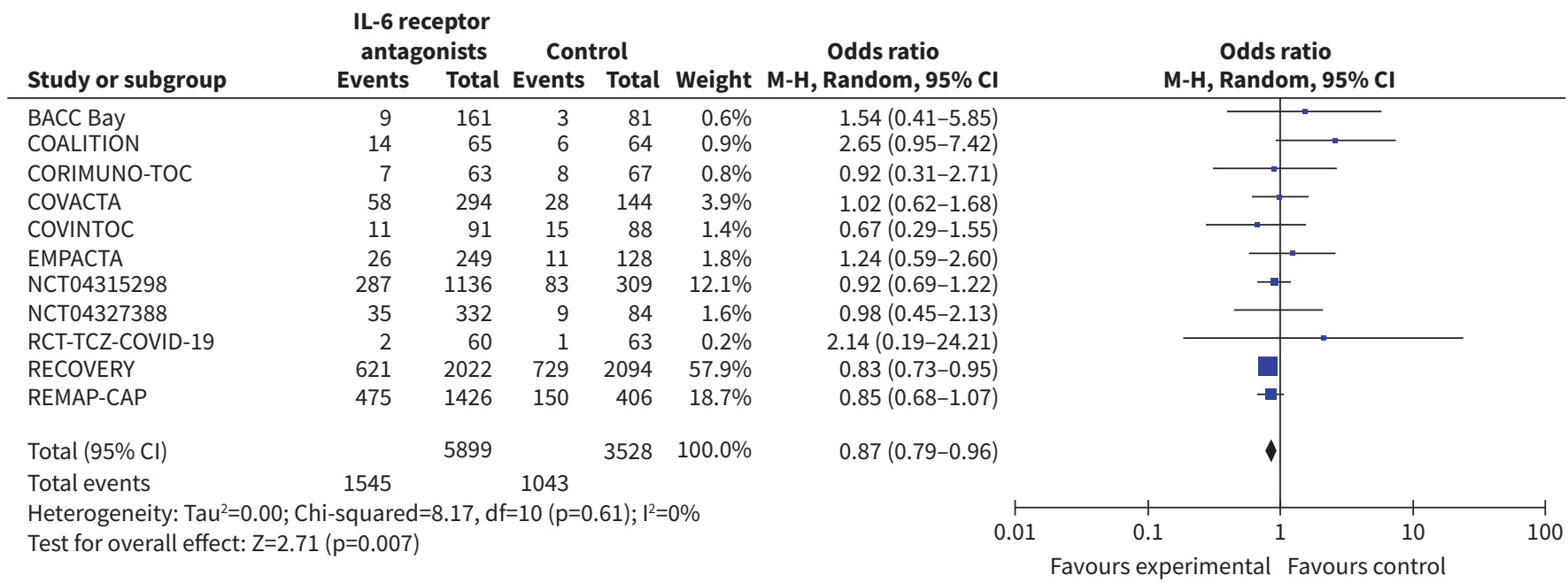

FIGURE 1 Forest plots showing the effects of corticosteroids and interleukin (IL)-6 receptor antagonists on mortality of hospitalised adult patients with coronavirus disease 2019. Study name has been used unless no name was available, in which case the trial registration identifier has been used. The weight represents the percentage contribution of each study to the summary effect estimate.

conducted in intensive care units and showed an effect estimate of 0.76 (0.55-1.05). The certainty of evidence was graded as moderate (supplementary table S1).

\section{IL-6 receptor antagonist monoclonal antibodies}

We identified 11 studies [18-28] with 9427 subjects, including two additional studies [18, 23] and two preprints [19, 24] following publication of the guidelines. Two reports of the REMAP-CAP trial were identified, reporting results for tocilizumab and sarilumab [19, 29]. It was established these were overlapping datasets containing data from the same subjects, and so the larger report from DerDE et al. [19] was included in the analysis. The original guidelines included one preprint and two preliminary reports, which have all been updated using finalised published data to complete this review [19, 21, 22]. SivAPALASINGAm et al. [24] (NCT04315298) reported results of a phase 2 and phase 3 programme for sarilumab in a single article. In addition, this article reported results for a dose of $200 \mathrm{mg}$ intravenous sarilumab and $400 \mathrm{mg}$ i.v. sarilumab. For the purposes of analysis, these were treated as a single trial and data for both doses pooled. Lescure et al. [23] (NCT04327388) also randomised patients to both $200 \mathrm{mg}$ and $400 \mathrm{mg}$ sarilumab doses, which were both pooled for analysis. This study presented data with mortality at both day 29 and day 60. Mortality at day 60 was used.

Overall, mortality in the treatment group was 1545 (26.2\%) out of 5899 and in the control group was 1043 (29.6\%) out of 3528. The pooled effect estimate was OR 0.87 (95\% CI 0.79-0.96, p=0.007), showing significant reduction in mortality with anti-IL-6 treatment. There was no heterogeneity $\left(\mathrm{I}^{2}=0 \%\right)$. The forest plot is shown in figure 1. It was noted that despite the overall significant effect, only one of the 11 trials showed statistically significant differences in favour of the intervention, the RECOVERY trial [21]. In 
terms of timing of treatment, the majority of studies were conducted on populations of patients outside the intensive care unit or a moderate-severe population including those receiving oxygen or ventilatory support. The largest study, the RECOVERY trial, showed a similar benefit in patients not receiving ventilator support (rate ratio $0.81,95 \%$ CI $0.67-0.99$ ) and receiving noninvasive ventilation (rate ratio 0.86, 95\% CI 0.74-1.00), with the suggestion of a lesser effect in those invasively ventilated at randomisation (rate ratio 0.93 , 95\% CI 0.74-1.18) [21].

As the review included data for both sarilumab and tocilizumab, a sub-analysis was conducted separately analysing the three sarilumab trials [19, 23, 24] and the nine tocilizumab trials [18-22, 25-28] (supplementary figure S3). One trial, REMAP-CAP, included both sarilumab and tocilizumab arms, and so is included in both analyses [19]. We found no meaningful difference in effect estimates between the two: sarilumab OR 0.88 (95\% CI 0.73-1.07, p=0.19) and tocilizumab OR 0.87 (95\% CI 0.78-0.96, p=0.009), resulting in a nonsignificant subgroup difference of $\mathrm{p}=0.89$. The certainty of evidence was graded as moderate (supplementary table S2).

\section{Hydroxychloroquine}

We identified 13 eligible studies [30-42] with 8721 subjects, including five additional published studies [31, 34-36, 42] and one preprint [39] following publication of the guidelines. Preprints used in the original analysis have been updated in this analysis [30,41]. Mortality in the treatment group was 628 (17.5\%) out of 3589, and in the control group was 984 (19.2\%) out of 5132. The pooled effect estimate was OR 1.09 (95\% CI $0.97-1.22, \mathrm{p}=0.14$ ) with no heterogeneity $\left(\mathrm{I}^{2}=0 \%\right)$. The forest plot is shown in figure 2. Four randomised controlled trials of hydroxychloroquine were excluded from our analysis due to the patient population being community based rather than hospital based [43-46]. The results from these studies showed no benefits from treatment in clinical status, with RÉA-NeTo et al. [44] identifying a significant worsening of clinical status, including an increased need for invasive mechanical ventilation relative risk (2.15, 95\% CI 1.05-4.40; p=0.030). ReIs et al. [43] stopped the hydroxychloroquine arm of their trial at first interim analysis due to futility based on data from another study. The certainty of evidence was graded as moderate (supplementary table S3).

\section{Azithromycin}

We identified five studies [33, 47-50] with 9067 subjects, including one additional study [50] and one preprint [47] following publication of the guidelines. The PRINCIPLE trial testing azithromycin in outpatients showed no evidence of benefit of azithromycin in nonhospitalised patients [51], but it is not included in the present meta-analysis focusing on hospitalised patients only. The ATOMIC2 trial by HINKs et al. [47] was included in the analysis, as patients were enrolled in hospital, although eligibility included the requirement to be eligible for ambulatory management. The COALITION study by CAVALCANTI et al. [33] compared azithromycin and hydroxychloroquine compared to hydroxychloroquine or standard care. Since hydroxychloroquine was widely used as standard care in many other studies included in the guidelines, the guideline panel determined that this study could be used to infer the efficacy of azithromycin. Including all studies mortality in the treatment group was 657 (20.7\%) out of 3169, and in the control group was 1250 (21.2\%) out of 5898. The pooled effect estimate was OR 0.97 (95\% CI 0.871.08, $\mathrm{p}=0.53$ ) with no heterogeneity $\left(\mathrm{I}^{2}=0 \%\right)$. Excluding COALITION and ATOMIC2, did not impact the conclusion (OR 0.97 95\% CI 0.87-1.08). The forest plot is shown in figure 2. The certainty of evidence was graded as moderate (supplementary table S4).

\section{Hydroxychloroquine and azithromycin in combination}

We identified two studies [33, 52] with 462 subjects, including one additional study [52] following publication of the guidelines and which was identified after the original search dates. Across these two studies, mortality in the treatment group was six (2.6\%) out of 233, and in the control group was eight (3.5\%) out of 229. The pooled effect estimate was OR 0.73 (95\% CI $0.25-2.15$, p=0.57) with no heterogeneity $\left(\mathrm{I}^{2}=0 \%\right)$. The forest plot is shown in figure 2 . The search identified one active comparator trial (HAHPS) comparing the efficacy of hydroxychloroquine versus azithromycin [53]; however, this study was not included in the analysis as it did not address any of the guideline PICO questions, but is discussed here. In common with other studies, the HAHPS study was stopped early due to futility. The data were assessed using a proportional odds modelling from a Bayesian perspective and concluded a $60 \%$ posterior probability that hydroxychloroquine was inferior to azithromycin. For the remaining two studies included in the meta-analysis the certainty of evidence was graded as low (supplementary table S5).

\section{Lopinavir/ritonavir}

We identified four studies [30, 42, 54, 55] with 8303 subjects, including one additional study [42] following publication of the guidelines. The DISCOVERY trial by ADER et al. [42] (an add-on trial to 


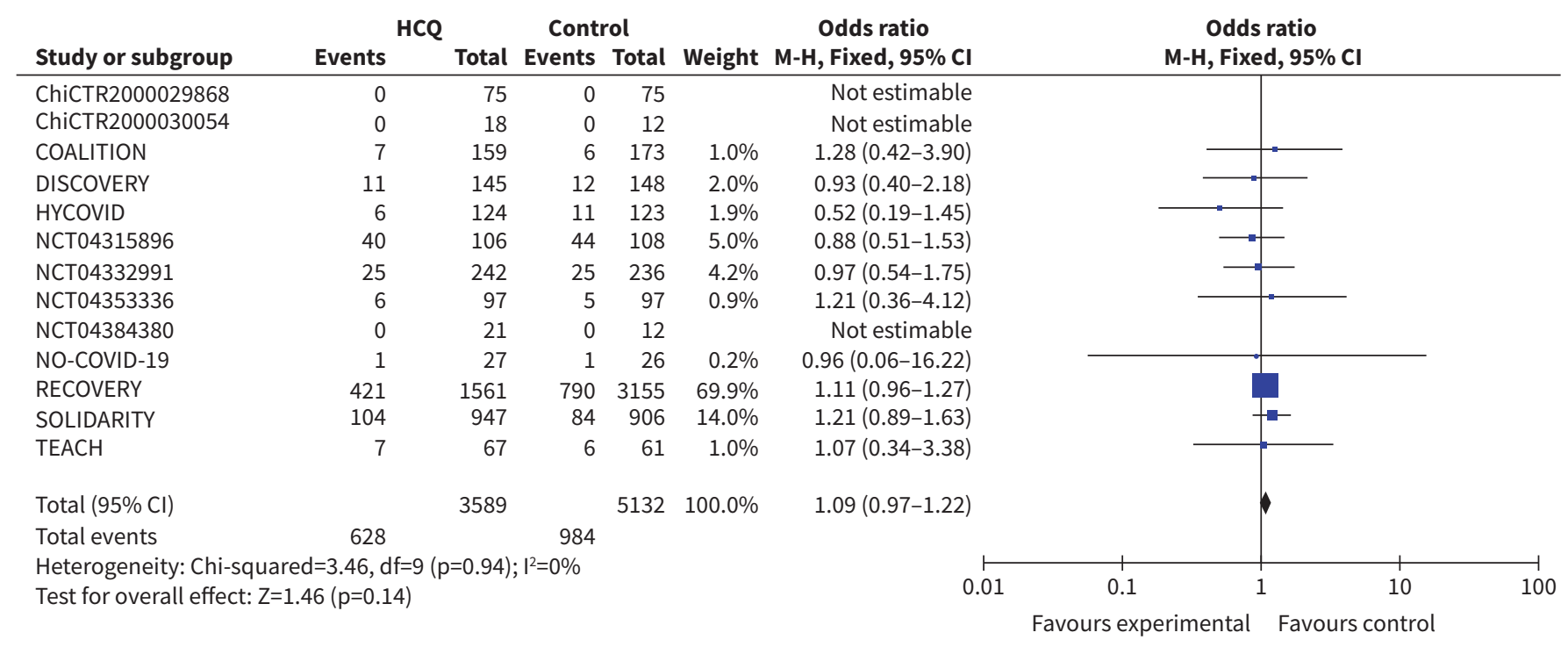

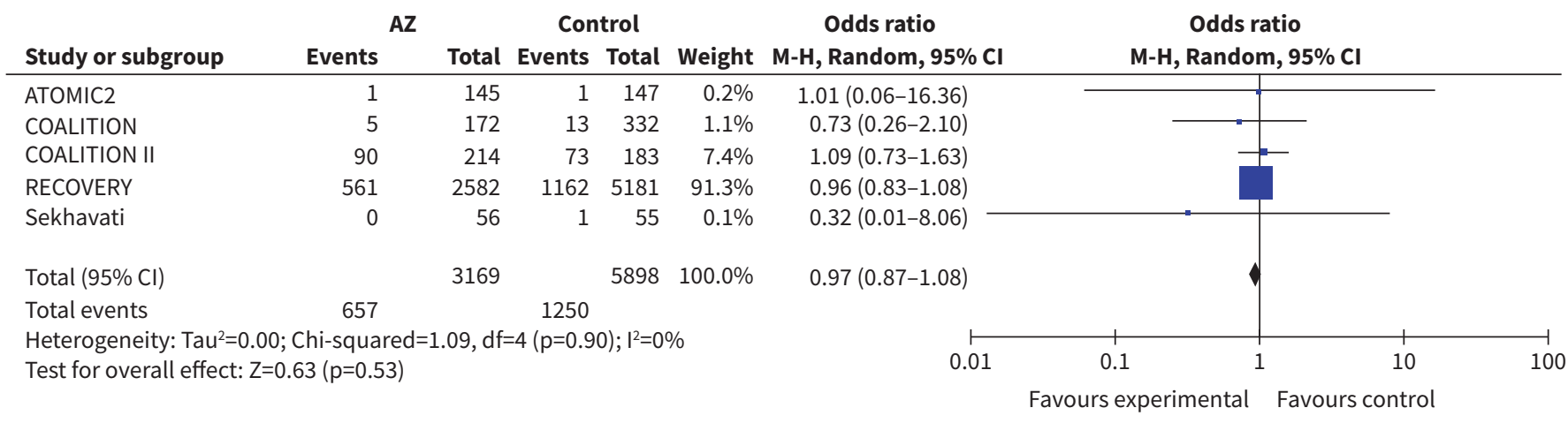

\begin{tabular}{|c|c|c|c|c|c|c|c|c|c|c|}
\hline \multirow[b]{2}{*}{ Study or subgroup } & \multicolumn{2}{|c|}{ HCQ and AZ } & \multicolumn{2}{|c|}{ Control } & \multirow[b]{2}{*}{ Weight } & Odds ratio & \multirow{2}{*}{\multicolumn{2}{|c|}{$\begin{array}{c}\text { Odds ratio } \\
\text { M-H, Fixed, } 95 \% \mathrm{Cl}\end{array}$}} & & \\
\hline & Events & Total & Events & Total & & M-H, Fixed, $95 \% \mathrm{Cl}$ & & & & \\
\hline Coalition & 5 & 172 & 6 & 173 & $73.9 \%$ & $0.83(0.25-2.78)$ & & - & & \\
\hline ProPAC-COVID & 1 & 61 & 2 & 56 & $26.1 \%$ & $0.45(0.04-5.10)$ & & & & \\
\hline Total $(95 \% \mathrm{Cl})$ & & 233 & & 229 & $100.0 \%$ & $0.73(0.25-2.15)$ & & & & \\
\hline Total events & 6 & & 8 & & & & & & & \\
\hline $\begin{array}{l}\text { Heterogeneity: Chi-sc } \\
\text { Test for overall effect }\end{array}$ & $\begin{array}{l}d=0.20, d \\
57(p=0.5\end{array}$ & $p=0.66)$ & $;\left.\right|^{2}=0 \%$ & & & 0.01 & 0.1 & & 10 & \\
\hline
\end{tabular}

FIGURE 2 Forest plots showing the effects of hydroxychloroquine (HCQ), azithromycin (AZ) and hydroxychloroquine in combination with azithromycin on mortality of hospitalised adult patients with coronavirus disease 2019. Study name has been used unless no name was available, in which case the trial registration identifier has been used. The weight represents the percentage contribution of each study to the summary effect estimate.

SOLIDARITY [30]) in which patients are randomly assigned either lopinavir/ritonavir, lopinavir/ritonavir/ interferon- $\beta 1$ a, hydroxychloroquine or remdesivir in comparison to standard of care in a 1:1:1:1 ratio, added another 293 hospitalised patients to the lopinavir/ritonavir analysis. The primary outcome was improved clinical status determined by a seven-point ordinal scale; however, due to treatment futility this arm and the hydroxychloroquine arm were stopped prematurely. No benefit to clinical status was observed from the data obtained (adjusted OR 0.83, 95\% CI 0.55-1.26; $\mathrm{p}=0.39$ ) and no impact was made on the overall mortality odds ratio published in the original guideline. Mortality in the treatment group was 555 (17.0\%) out of 3259, and in the control group was 950 (18.8\%) out of 5044. The pooled effect estimate was OR 1.02 (95\% CI $0.91-1.15, \mathrm{p}=0.72)$ with no heterogeneity $\left(\mathrm{I}^{2}=0 \%\right)$. The forest plot is shown in figure 3 . The certainty of evidence was graded as high (supplementary table S6). 


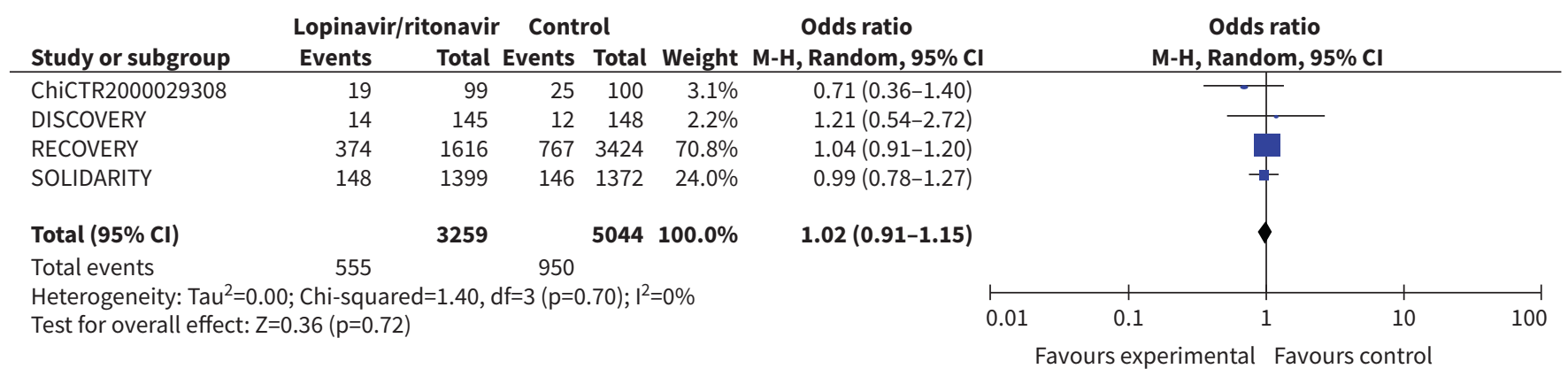

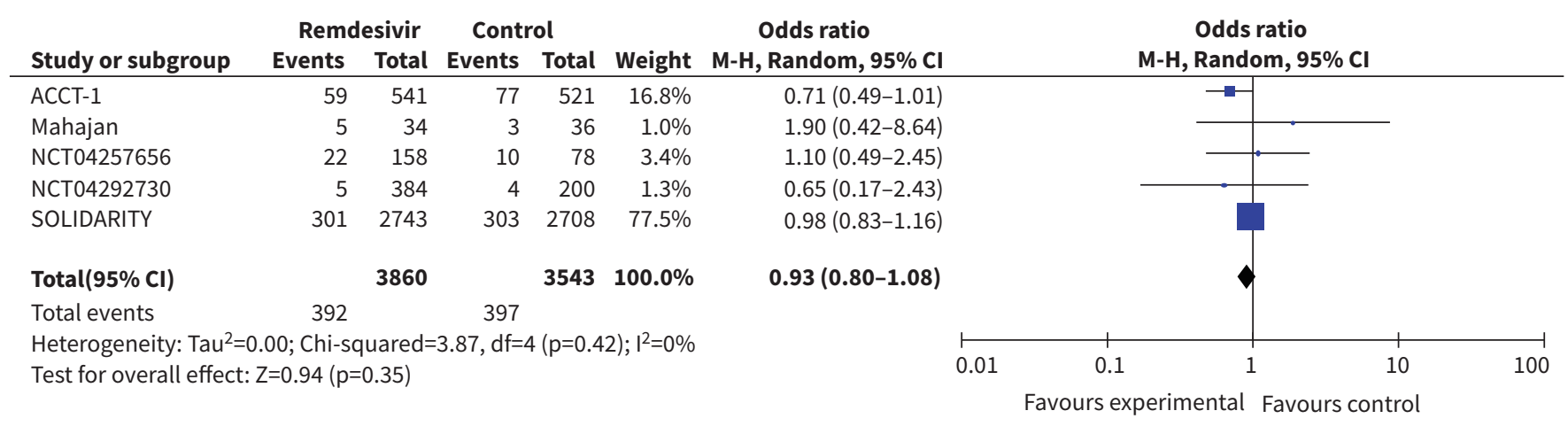

FIGURE 3 Forest plots showing the effects of lopinavir/ritonavir and remdesivir on mortality of hospitalised adult patients with coronavirus disease 2019. Study name has been used unless no name was available, in which case the trial registration identifier has been used. The weight represents the percentage contribution of each study to the summary effect estimate.

\section{Remdesivir}

We identified five studies meeting the inclusion criteria [30, 56-59] with 7403 subjects, including one additional study [59] following publication of the guidelines. Mortality in the treatment group was 392 (10.2\%) out of 3860, and in the control group was $397(11.2 \%)$ out of 3543. The pooled effect estimate was OR 0.93 (95\% CI $0.80-1.08, \mathrm{p}=0.35)$ with no heterogeneity $\left(\mathrm{I}^{2}=0 \%\right)$. The forest plot is shown in figure 3. No recommendation was made regarding the use of remdesivir in the original guidelines [8]. A randomised, open-label trial excluded from our analysis due to having no appropriate comparator treatment assessed the differential benefits of receiving a 5-day course of remdesivir treatment $(\mathrm{n}=200)$ versus receiving a 10-day course of treatment $(n=197)$ in hospitalised patients. The results showed no statistically significant difference between the two treatment durations [60]. The certainty of evidence was graded as moderate (supplementary table S7).

\section{Colchicine}

We identified three studies [61-63] with 11517 subjects, including one preprint [63] following publication of the guidelines. The COLCORONA trial showed no statistically significant benefit of colchicine in outpatients with COVID-19, but was not included as this analysis focused on hospitalised patients only [64]. The interim analysis from Lopes et al. [61], which was included in the original guidelines, was updated to reflect the data from all trial participants (increasing participant number from 35 (18 placebo versus 17 colchicine) to 72 (36 placebo versus 36 colchicine)). Mortality in the treatment group was 1174 (20.6\%) out of 5701 and 1196 (20.6\%) out of 5816 in the control group. The pooled effect estimate was OR 0.64 (95\% CI 0.22-1.89, $\mathrm{p}=0.42)$, but with moderate heterogeneity $\left(\mathrm{I}^{2}=34 \%\right)$. The two smaller studies, which were rated as high risk of bias in the original guideline document showed benefits in favour of colchicine [61, 62], while the much larger RECOVERY trial found no evidence of benefit OR 1.01 (95\% CI 0.92-1.10) [63]. The forest plot is shown in figure 4. The certainty of evidence was graded as low (supplementary table S8).

\section{Interferon- $\beta$}

We identified four studies [30, 65-67] with 4307 subjects, including one additional study [67] following publication of the guidelines. Alavi DarazAm et al. [67] performed a three-arm study randomly assigning a 


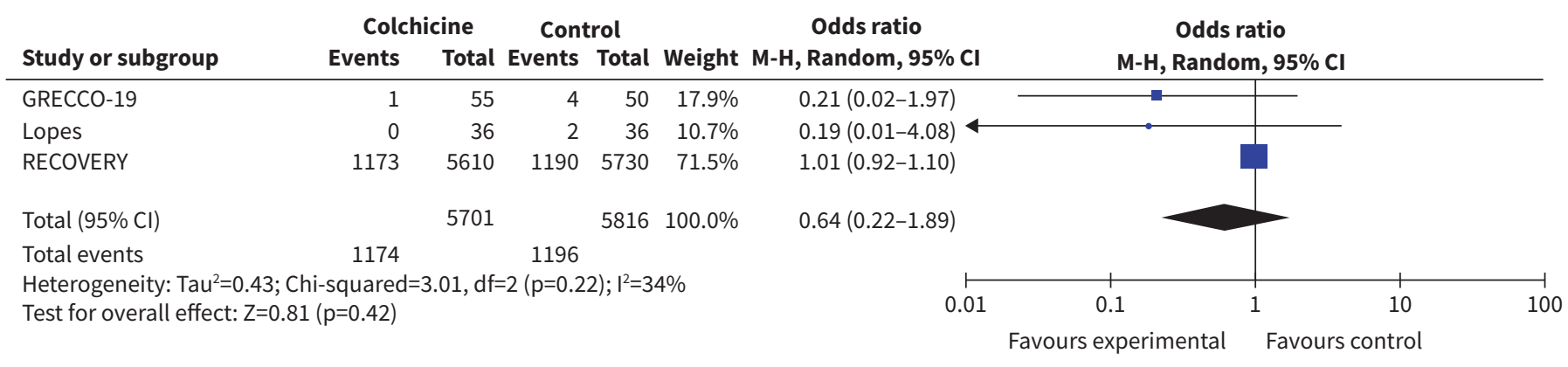

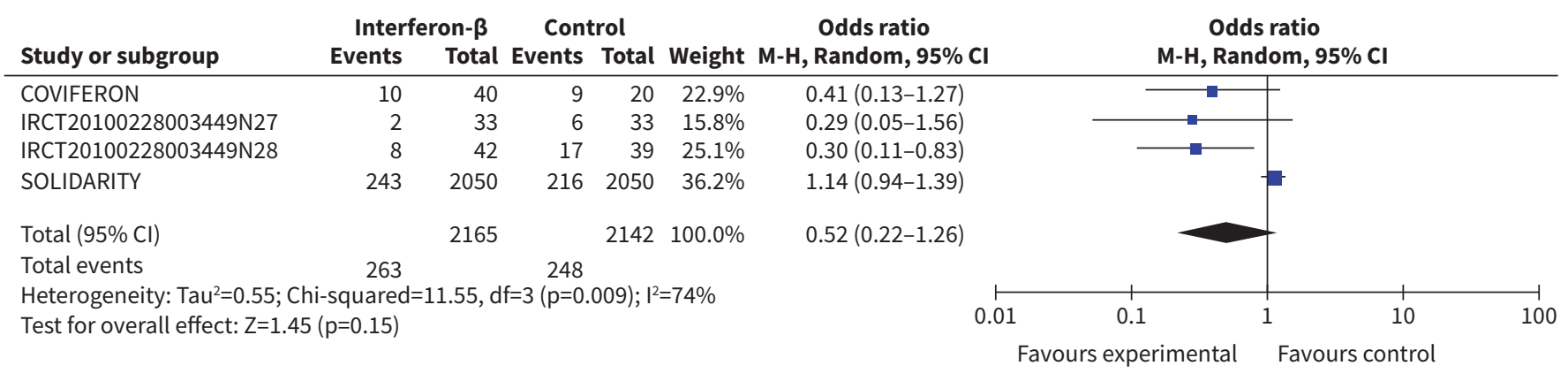

FIGURE 4 Forest plots showing the effects of colchicine and interferon- $\beta$ on mortality of hospitalised adult patients with coronavirus disease 2019. Study name has been used unless no name was available, in which case the trial registration identifier has been used. The weight represents the percentage contribution of each study to the summary effect estimate.

1:1:1 ratio of systemic interferon- $\beta 1 \mathrm{a}$, interferon- $\beta 1 \mathrm{~b}$ and a control group. The primary end-point for this study was time to clinical improvement as defined by a seven-step ordinal scale or discharge. In this study, interferon- $\beta 1$ a showed a significant improvement in time to clinical improvement when compared to control (hazard ratio (HR) 2.36, 95\% CI 1.10-5.17; p=0.031), whereas interferon- $\beta 1 \mathrm{~b}$ did not (HR 1.42, 95\% CI 0.63-3.16; p=0.395). The largest study (SOLIDARITY) included in the meta-analysis showed no significant benefit, with a trend towards harm [30]. For the purpose of this analysis looking at mortality outcome, data from arms containing interferon- $\beta 1 \mathrm{a}$ and interferon- $\beta 1 \mathrm{~b}$ were combined. Mortality in the treatment group was $263(12.1 \%)$ out of 2165 and 248 (11.6\%) out of 2142 in the control group. The pooled effect estimate was OR 0.52 (95\% CI 0.22-1.26, $\mathrm{p}=0.15$ ). The forest plot is shown in figure 4. A study of nebulised interferon- $\beta 1$ a was identified [68]. This trial randomised 50 patients to nebulised interferon and 51 patients to placebo and demonstrated a statistically significant improvement in clinical status (OR 2.32, 95\% CI 1.07-5.04; p=0.033). It was not considered appropriate to pool nebulised and systemic interferon data, and therefore this trial is not included in the meta-analysis. The certainty of evidence was graded as low (supplementary table S9).

\section{Discussion}

Mortality is the critical outcome for testing the efficacy of therapeutics in hospitalised patients with COVID-19, a condition with a high mortality rate globally [1]. This systematic review and meta-analysis confirms that corticosteroids and anti-IL-6 receptor monoclonal antibodies reduce mortality in hospitalised patients with COVID-19. The sub-analyses with different corticosteroids suggest no class effect, as there were no subgroup differences between dexamethasone, hydrocortisone and methylprednisolone. Further studies will be required to establish the most appropriate dose and duration of therapy. In terms of the anti-IL-6 receptor monoclonal antibodies, it is also noted that while the majority of published evidence concerns tocilizumab, there were no significant subgroup differences, suggesting that sarilumab can be considered equally efficacious. For corticosteroids, the greatest benefits were observed in patients requiring mechanical ventilation with significant benefits observed in patients requiring oxygen, but no benefits in those not requiring oxygen. For anti-IL-6 receptor monoclonal antibodies, the majority of studies were performed in patients requiring oxygen or noninvasive ventilation with reduced mortality observed in these groups. 


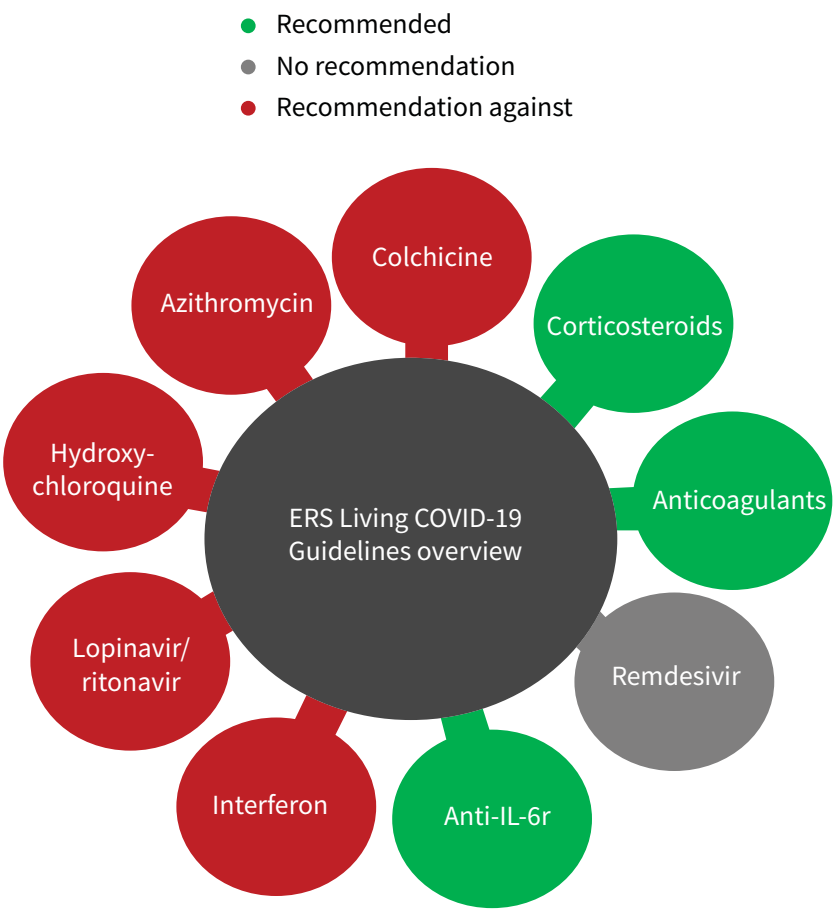

FIGURE 5 Summary of recommendations from the European Respiratory Society (ERS) guidelines for hospitalised patients with coronavirus disease 2019 (COVID-19).

We found no mortality benefit of hydroxychloroquine or azithromycin, or the combination of hydroxychloroquine and azithromycin, lopinavir/ritonavir, remdesivir, colcichine or interferon- $\beta$. These results are consistent with the earlier results included in the ERS guidelines and support the original recommendations regarding these agents included in the guidelines. A summary of the guideline recommendations is shown in figure 5.

As the medical science community continues to work relentlessly on identifying the best treatment methods in COVID-19, the resulting literature is published at an unprecedented rate, meaning that guidelines are "out of date" at the time of their publication. To minimise the disconnect between new evidence and recommendations, "living guidelines" allow for frequent, rapid literature searches to identify where new evidence means new practice-changing evidence. These important updates can then be published immediately and communicated to the appropriate communities much faster than a standard guideline, which is typically updated every 3-4 years.

Our update to the systematic review and meta-analysis has seen the addition of 20 randomised controlled trials published since January 2021. The trials include an additional 25171 hospitalised adult patients with COVID-19. As described in the original guidelines, the main aim in managing hospitalised patients is to reduce mortality and prevent worsening of health status from the point of hospitalisation. Mortality is a priority outcome, due to the high mortality rate of COVID-19 in combination with the high reproductive rate $\left(\mathrm{R}_{0}\right)$ of SARS-CoV-2, estimated as 2.5 [69].

Our results extend the results of the original ERS guidelines in which IL-6 receptor antagonist monoclonal antibodies had not accumulated sufficient evidence to show a significant effect on mortality, but with the additional literature a significant benefit is demonstrated in the present analysis.

It was well documented at the start of the pandemic that hydroxychloroquine was the treatment most widely studied, and therefore unsurprisingly, the largest number of trials we identified were for this agent. A meta-analysis performed by AxFons et al. [70] found that the use of hydroxychloroquine was statistically detrimental (OR 1.11, 95\% CI 1.02-1.2; p=0.02). Unlike AxFons et al., we did not include prophylaxis trials or unpublished data; our findings from hospitalised patients showed a very similar trend, but with wider confidence intervals (OR 1.10, 95\% CI 0.97-1.23; p=0.53). With the addition of five new hydroxychloroquine trials which include almost 1000 patients further confirming the detrimental effects of 
hydroxychloroquine use in COVID-19 patients, the ERS guideline panel has recommended that there is no need to continue studying this treatment as a potential therapy in this population. Future iterations of the ERS guidelines will not address hydroxychloroquine. $>8000$ patients were added to the azithromycin analysis, with the vast majority taking part in the RECOVERY platform trial; however, this did not show any beneficial evidence for azithromycin use in COVID-19 management and this treatment will not be updated in future guidelines. No improvements were observed from therapeutic use of lopinavir/ritonavir, and this too will not be updated in future guidelines. It is acknowledged that there is a need to add new drugs for which there are recently published trials including antibodies, convalescent plasma, baricitinib and anti-granulocyte-macrophage colony-stimulating factor, and these will feature in future guidelines as further data accumulates. In addition, new randomised controlled trial data have been published in recent weeks showing that therapeutic anticoagulation may be beneficial in hospitalised patients outside the intensive care unit [71] and that continuous positive airway pressure is superior to conventional oxygen therapy in patients requiring ventilatory support [72]. These results will be incorporated into the next version of the treatment guidelines.

We acknowledge there are limitations to this study. As the pandemic continues, reports show higher proportions of children and adolescents becoming infected with COVID-19 and our search covers only the adult population. Our search has remained in the hospitalised population with the focus on mortality outcome; however, many countries have implemented successful vaccination programmes and as a result fewer people are being admitted to hospital with severe SARS-CoV-2 infection. As the impact of COVID-19 changes, the research needs to be flexible and reflect this with more studies needed in the community, inclusive of the younger population and with consideration of new outcomes such as prevention and reduction of long-COVID symptoms.

Conclusion

Corticosteroids and anti-IL-6 receptor monoclonal antibodies reduce mortality in COVID-19 patients.

Provenance: Commissioned article, peer reviewed.

Acknowledgements: The members of the European Respiratory Society COVID-19 Guidelines Task Force are: Bin Cao, Marc Humbert, Michal Shteinberg, Katerina M. Antoniou, Charlotte Suppli Ulrik, Helen Parks, Chen Wang, Jieming Qu, Daiana Stolz, Christopher Brightling, Tobias Welte, Stefano Aliberti and Anita K. Simonds.

Conflict of interest: M.L. Crichton has nothing to disclose. P.C. Goeminne has nothing to disclose. K. Tuand has nothing to disclose. T. Vandendriessche has nothing to disclose. T. Tonia is an ERS methodologist. N. Roche reports grants and personal fees from Boehringer Ingelheim, Novartis and Pfizer, personal fees from GSK, AstraZeneca, Chiesi, Sanofi and Zambon, outside the submitted work. J.D. Chalmers reports grants and personal fees from AstraZeneca, Boehringer Ingelheim, GlaxoSmithKline and Insmed, grants from Gilead Sciences, personal fees from Chiesi, Novartis and Zambon, outside the submitted work.

Support statement: Funded by the European Respiratory Society. Funding information for this article has been deposited with the Crossref Funder Registry.

References

1 da Costa VG, Moreli ML, Saivish MV. The emergence of SARS, MERS and novel SARS-2 coronaviruses in the 21st century. Arch Virol 2020; 165: 1517-1526.

2 World Health Organization. Weekly Epidemiological Update on COVID-19 - 29 June 2021. 2021. Available from: www.who.int/publications/m/item/weekly-epidemiological-update-on-covid-19---29-june-2021/

3 Vittoria BL, Imbesi C, Irene G, et al. New approaches and repurposed antiviral drugs for the treatment of the SARS-CoV-2 infection. Pharmaceuticals 2021; 14: 503.

4 Janssen DJA, Ekström M, Currow DC, et al. COVID-19: guidance on palliative care from a European Respiratory Society international task force. Eur Respir J 2020; 56: 2002583.

5 Spruit MA, Holland AE, Singh SJ, et al. COVID-19: interim guidance on rehabilitation in the hospital and post-hospital phase from a European Respiratory Society and American Thoracic Society-coordinated international task force. Eur Respir J 2020; 56: 2002197.

6 Bai C, Chotirmall SH, Rello J, et al. Updated guidance on the management of COVID-19: from an American Thoracic Society/European Respiratory Society coordinated international task force (29 July 2020). Eur Respir Rev 2020; 29: 200287.

7 Alonso-Coello P, Oxman AD, Moberg J, et al. Marcos GRADE de la evidencia a la decisión (EtD): un enfoque sistemático y transparente para tomar decisiones sanitarias bien informadas. 2: Guías de práctica clínica. 
[GRADE Evidence to Decision (EtD) frameworks: a systematic and transparent approach to making well informed healthcare choices. 2: Clinical practice guidelines]. Gac Sanit 2018; 32: 167.e1-167.

8 Chalmers JD, Crichton ML, Goeminne PC, et al. Management of hospitalised adults with coronavirus disease-19 (COVID-19): a European Respiratory Society living guideline. Eur Respir J 2021; 57: 2100048.

9 Edalatifard M, Akhtari M, Salehi M, et al. Intravenous methylprednisolone pulse as a treatment for hospitalised severe COVID-19 patients: results from a randomised controlled clinical trial. Eur Respir J 2020; 56: 2002808.

10 Dequin PF, Heming N, Meziani F, et al. Effect of hydrocortisone on 21-day mortality or respiratory support among critically ill patients with COVID-19: a randomized clinical trial. JAMA 2020; 324: 1298-1306.

11 Angus DC, Derde L, Al-Beidh F, et al. Effect of hydrocortisone on mortality and organ support in patients with severe COVID-19: the REMAP-CAP COVID-19 corticosteroid domain randomized clinical trial. JAMA 2020; 324 1317-1329.

12 Tomazini BM, Maia IS, Cavalcanti AB, et al. Effect of dexamethasone on days alive and ventilator-free in patients with moderate or severe acute respiratory distress syndrome and COVID-19: the CoDEX randomized clinical trial. JAMA 2020; 324: 1307-1316.

13 Jamaati $\mathrm{H}$, Hashemian SM, Farzanegan B, et al. No clinical benefit of high dose corticosteroid administration in patients with COVID-19: a preliminary report of a randomized clinical trial. Eur $J$ Pharmacol 2021; 897: 173947.

14 Corral-Gudino L, Bahamonde A, Arnaiz-Revillas F, et al. Methylprednisolone in adults hospitalized with COVID-19 pneumonia: an open-label randomized trial (GLUCOCOVID). Wien Klin Wochenschr 2021; 133: 303-311.

15 Jeronimo CMP, Farias MEL, Val FFA, et al. Methylprednisolone as adjunctive therapy for patients hospitalized with coronavirus disease 2019 (COVID-19; Metcovid): a randomized, double-blind, phase IIb, placebo-controlled trial. Clin Infect Dis 2021; 72: e373-e381.

16 Horby P, Lim WS, Emberson JR, et al. Dexamethasone in hospitalized patients with covid-19. N Engl J Med 2021; 384: 693-704.

17 Tang X, Feng YM, Ni JX, et al. Early use of corticosteroid may prolong SARS-CoV-2 shedding in non-intensive care unit patients with COVID-19 pneumonia: a multicenter, single-blind, randomized control trial. Respiration 2021; 100: 116-126.

18 Soin AS, Kumar K, Choudhary NS, et al. Tocilizumab plus standard care versus standard care in patients in India with moderate to severe COVID-19-associated cytokine release syndrome (COVINTOC): an open-label, multicentre, randomised, controlled, phase 3 trial. Lancet Respir Med 2021; 9: 511-521.

19 Derde LPG, Gordon AC, Mouncey PR, et al. Effectiveness of tocilizumab, sarilumab, and anakinra for critically ill patients with COVID-19. The REMAP-CAP COVID-19 immune modulation therapy domain randomized clinical trial. medRxiv 2021; preprint [https://doi.org/10.1101/2021.06.18.21259133].

20 Stone JH, Frigault MJ, Serling-Boyd NJ, et al. Efficacy of tocilizumab in patients hospitalized with covid-19. N Engl J Med 2020; 383: 2333-2344.

21 Abani O, Abbas A, Abbas F, et al. Tocilizumab in patients admitted to hospital with COVID-19 (RECOVERY): a randomised, controlled, open-label, platform trial. Lancet 2021; 397: 1637-1645.

22 Rosas 10, Bräu N, Waters M, et al. Tocilizumab in hospitalized patients with severe Covid-19 pneumonia. N Engl J Med 2021; 384: 1503-1516.

23 Lescure FX, Honda H, Fowler RA, et al. Sarilumab in patients admitted to hospital with severe or critical COVID-19: a randomised, double-blind, placebo-controlled, phase 3 trial. Lancet Respir Med 2021; 9: 522-532.

24 Sivapalasingam S, Lederer D, Bhore R, et al. A randomized placebo-controlled trial of sarilumab in hospitalized patients with covid-19. medRxiv 2021; preprint [https://doi.org/10.1101/2021.05.13.21256973].

25 Hermine $\mathrm{O}$, Mariette X, Tharaux PL, et al. Effect of tocilizumab vs usual care in adults hospitalized with COVID-19 and moderate or severe pneumonia: a randomized clinical trial. JAMA Intern Med 2021; 181: 32-40.

26 Salama C, Han J, Yau L, et al. Tocilizumab in patients hospitalized with Covid-19 pneumonia. N Engl J Med 2021; 384: 20-30.

27 Salvarani C, Dolci G, Massari M, et al. Effect of tocilizumab vs standard care on clinical worsening in patients hospitalized with COVID-19 pneumonia: a randomized clinical trial. JAMA Intern Med 2021; 181: 24-31.

28 Veiga VC, Prats JAGG, Farias DLC, et al. Effect of tocilizumab on clinical outcomes at 15 days in patients with severe or critical coronavirus disease 2019: randomised controlled trial. BMJ 2021; 372: n84

29 Gordon AC, Mouncey PR, Al-Beidh F, et al. Interleukin-6 receptor antagonists in critically ill patients with covid-19. N Engl J Med 2021; 384: 1491-1502.

30 Pan H, Peto R, Henao-Restrepo AM, et al. Repurposed antiviral drugs for Covid-19 - interim WHO Solidarity Trial results. N Engl J Med 2021; 384: 497-511.

31 Ulrich RJ, Troxel AB, Carmody E, et al. Treating COVID-19 with hydroxychloroquine (TEACH): a multicenter, double-blind randomized controlled trial in hospitalized patients. Open Forum Infect Dis 2020; 7: ofaa446.

32 Abd-Elsalam S, Esmail ES, Khalaf M, et al. Hydroxychloroquine in the treatment of COVID-19: a multicenter randomized controlled study. Am J Trop Med Hyg 2020; 103: 1635-1639. 
33 Cavalcanti $A B$, Zampieri FG, Rosa RG, et al. Hydroxychloroquine with or without azithromycin in mild-to-moderate covid-19. N Engl J Med 2020; 383: 2041-2052.

34 Self WH, Semler MW, Leither LM, et al. Effect of hydroxychloroquine on clinical status at 14 days in hospitalized patients with COVID-19: a randomized clinical trial. JAMA 2020; 324: 2165-2176.

35 Dubée V, Roy P-M, Vielle B, et al. Hydroxychloroquine in mild-to-moderate coronavirus disease 2019: a placebo-controlled double blind trial. Clin Microbiol Infect 2021; 27: 1124-1130.

36 Lyngbakken MN, Berdal JE, Eskesen A, et al. A pragmatic randomized controlled trial reports lack of efficacy of hydroxychloroquine on coronavirus disease 2019 viral kinetics. Nat Commun 2020; 11: 6-11.

37 Horby PW, Mafham M, Linsell L, et al. Effect of hydroxychloroquine in hospitalized patients with COVID-19: preliminary results from a multi-centre, randomized, controlled trial. MedRxiv 2020; preprint [https://doi.org/ 10.1101/2020.07.15.20151852].

38 Tang W, Cao Z, Han M, et al. Hydroxychloroquine in patients with mainly mild to moderate coronavirus disease 2019: open label, randomised controlled trial. BMJ 2020; 369: m1849.

39 Hernandez-Cardenas C, Thirion-Romero I, Rivera-Martinez NE, et al. Hydroxychloroquine for the treatment of severe respiratory infection by COVID-19: a randomized controlled trial. medRxiv 2021; preprint [https://doi. org/10.1101/2021.02.01.21250371].

40 Chen L, Zhang Z-Y, Fu J-G, et al. Efficacy and safety of chloroquine or hydroxychloroquine in moderate type of COVID-19: a prospective open-label randomized controlled study. medRxiv 2020; preprint [https://doi.org/ 10.1101/2020.06.19.20136093].

41 Chen $\mathrm{C}-\mathrm{P}$, Lin Y-C, Chen T-C, et al. A multicenter, randomized, open-label, controlled trial to evaluate the efficacy and tolerability of hydroxychloroquine and a retrospective study in adult patients with mild to moderate coronavirus disease 2019 (COVID-19). PLoS One 2020; 15: e0242763.

42 Ader F, Peiffer-Smadja N, Poissy J, et al. An open-label randomized controlled trial of the effect of lopinavir/ ritonavir, lopinavir/ritonavir plus IFN- $\beta$-1a and hydroxychloroquine in hospitalized patients with COVID-19. Clin Microbiol Infect 2021; 27: 1826-1837.

43 Reis G, Moreira Silva EADS, Medeiros Silva DC, et al. Effect of early treatment with hydroxychloroquine or lopinavir and ritonavir on risk of hospitalization among patients with COVID-19: the TOGETHER randomized clinical trial. JAMA Netw Open 2021; 4: e216468.

44 Réa-Neto Á, Bernardelli RS, Câmara BMD, et al. An open-label randomized controlled trial evaluating the efficacy of chloroquine/hydroxychloroquine in severe COVID-19 patients. Sci Rep 2021; 11: 9023.

45 Johnston C, Brown ER, Stewart J, et al. Hydroxychloroquine with or without azithromycin for treatment of early SARS-CoV-2 infection among high-risk outpatient adults: a randomized clinical trial. EClinicalMedicine 2021; 33: 100773.

46 Purwati Budiono Rachman BE, et al. A randomized, double-blind, multicenter clinical study comparing the efficacy and safety of a drug combination of lopinavir/ritonavir-azithromycin, lopinavir/ritonavir-doxycycline, and azithromycin-hydroxychloroquine for patients diagnosed with mild to moderate COVID-19 infections. Biochem Res Int 2021; 2021: 6685921.

47 Hinks TSC, Cureton L, Knight R, et al. A randomised clinical trial of azithromycin versus standard care in ambulatory COVID-19 - the ATOMIC2 trial. SSRN 2021; preprint [http://dx.doi.org/10.2139/ssrn.3827019].

48 Sekhavati E, Jafari F, SeyedAlinaghi $S$, et al. Safety and effectiveness of azithromycin in patients with COVID-19: an open-label randomised trial. Int J Antimicrob Agents 2020; 56: 106143.

49 Furtado RHM, Berwanger O, Fonseca HA, et al. Azithromycin in addition to standard of care versus standard of care alone in the treatment of patients admitted to the hospital with severe COVID-19 in Brazil (COALITION II): a randomised clinical trial. Lancet 2020; 396: 959-967.

50 Abaleke E, Abbas M, Abbasi S, et al. Azithromycin in patients admitted to hospital with COVID-19 (RECOVERY): a randomised, controlled, open-label, platform trial. Lancet 2021; 397: 605-612.

51 Butler CC, Dorward J, Yu LM, et al. Azithromycin for community treatment of suspected COVID-19 in people at increased risk of an adverse clinical course in the UK (PRINCIPLE): a randomised, controlled, open-label, adaptive platform trial. Lancet 2021; 397: 1063-1074.

52 Sivapalan P, Suppli Ulrik C, Lapperre TS, et al. Azithromycin and hydroxychloroquine in hospitalised patients with confirmed COVID-19 - a randomised double-blinded placebo-controlled trial. Eur Respir J 2021; 2100752.

53 Brown SM, Peltan I, Kumar N, et al. Hydroxychloroquine versus azithromycin for hospitalized patients with COVID-19: results of a randomized, active comparator trial. Ann Am Thorac Soc 2021; 18: 590-597.

54 Cao B, Wang Y, Wen D, et al. A trial of lopinavir-ritonavir in adults hospitalized with severe covid-19. N Engl J Med 2020; 382: 1787-1799.

55 Horby PW, Mafham M, Bell JL, et al. Lopinavir-ritonavir in patients admitted to hospital with COVID-19 (RECOVERY): a randomised, controlled, open-label, platform trial. Lancet 2020; 396: 1345-1352.

56 Spinner CD, Gottlieb RL, Criner GJ, et al. Effect of remdesivir vs standard care on clinical status at 11 days in patients with moderate COVID-19: a randomized clinical trial. JAMA 2020; 324: 1048-1057.

57 Wang Y, Zhang D, Du G, et al. Remdesivir in adults with severe COVID-19: a randomised, double-blind, placebo-controlled, multicentre trial. Lancet 2020; 395: 1569-1578. 
58 Beigel JH, Tomashek KM, Dodd LE, et al. Remdesivir for the treatment of covid-19 - final report. N Engl J Med 2020; 383: 1813-1826.

59 Mahajan L, Singh AP, Gifty. Clinical outcomes of using remdesivir in patients with moderate to severe COVID-19: a prospective randomised study. Ind J Anaesth 2021; 65: S41-S46.

60 Goldman JD, Lye DCB, Hui DS, et al. Remdesivir for 5 or 10 days in patients with severe covid-19. N Engl J Med 2020; 383: 1827-1837.

61 Lopes MI, Bonjorno LP, Giannini MC, et al. Beneficial effects of colchicine for moderate to severe COVID-19: a randomised, double-blinded, placebo-controlled clinical trial. RMD Open 2021; 7: e001455.

62 Deftereos SG, Giannopoulos G, Vrachatis DA, et al. Effect of colchicine vs standard care on cardiac and inflammatory biomarkers and clinical outcomes in patients hospitalized with coronavirus disease 2019: the GRECCO-19 randomized clinical trial. JAMA Netw Open 2020; 3: e2013136.

63 Horby PW, Campbell M, Spata E, et al. Colchicine in patients admitted to hospital with COVID-19 (RECOVERY): a randomised, controlled, open-label, platform trial. medRxiv 2021; preprint [https://doi.org/10.1101/2021.05 18.21257267].

64 Tardif J-C, Bouabdallaoui N, L'Allier PL, et al. Colchicine for community-treated patients with COVID-19 (COLCORONA): a phase 3, randomised, double-blinded, adaptive, placebo-controlled, multicentre trial. Lancet Respir Med 2021; 9: 924-932.

65 Davoudi-Monfared E, Rahmani $\mathrm{H}$, Khalili $\mathrm{H}$, et al. A randomized clinical trial of the efficacy and safety of interferon $\beta$-1a in treatment of severe COVID-19. Antimicrob Agents Chemother 2020; 64: e01061-20.

66 Rahmani H, Davoudi-Monfared E, Nourian A, et al. Interferon $\beta$-1b in treatment of severe COVID-19: a randomized clinical trial. Int Immunopharmacol 2020; 88: 106903.

67 Alavi Darazam I, Shokouhi S, Pourhoseingholi MA, et al. Role of interferon therapy in severe COVID-19: the COVIFERON randomized controlled trial. Sci Rep 2021; 11: 8059.

68 Monk PD, Marsden RJ, Tear VJ, et al. Safety and efficacy of inhaled nebulised interferon beta-1a (SNG001) for treatment of SARS-CoV-2 infection: a randomised, double-blind, placebo-controlled, phase 2 trial. Lancet Respir Med 2021; 9: 196-206.

69 Petersen E, Koopmans M, Go U, et al. Comparing SARS-CoV-2 with SARS-CoV and influenza pandemics. Lancet Infect Dis 2020; 20: e238-e244.

70 Axfors C, Schmitt AM, Janiaud P, et al. Mortality outcomes with hydroxychloroquine and chloroquine in COVID-19 from an international collaborative meta-analysis of randomized trials. Nat Commun 2021; 12: 2349.

71 Lawler PR, Goligher EC, Berger JS, et al. Therapeutic anticoagulation with heparin in noncritically ill patients with covid-19. N Engl J Med 2021; 385: 790-802.

72 Perkins GD, Ji C, Connolly BA, et al. An adaptive randomized controlled trial of non-invasive respiratory strategies in acute respiratory failure patients with COVID-19. medRxiv 2021; preprint [https://doi.org/10.1101/ 2021.08.02.21261379]. 